\title{
Update on the management of early-stage breast cancer
}

Ghaith Bahjat Heilat, Meagan E Brennan, James French

\section{Background}

Breast cancer affects one in eight Australian women. While surgery, chemotherapy, radiotherapy and endocrine therapy are still the main treatments, there have been changes in the sequencing of treatment and advances in each therapy. The general practitioner (GP) is involved at each stage of the patient's journey.

\section{Objective}

This article discusses the current approach to the management of early breast cancer. It focuses on changes in recent years and discusses the role of the GP in supporting women in their decision-making and treatment.

\section{Discussion}

Key changes include the increasing use of neoadjuvant chemotherapy, the development of advanced oncoplastic surgery and breast reconstruction techniques, the use of gene expression profiling and the recommendation for extended adjuvant endocrine therapy for up to 10 years.
IN AUSTRALIA, BREAST CANCER is the most common cancer in women, affecting one in eight women by the age of $80 .^{1,2}$ Breast cancer incidence is now stable and mortality is decreasing, ${ }^{3}$ due partly to early diagnosis and partly to advances in treatment. In the area of treatment, multidisciplinary care remains important as there are different options for sequencing of treatment, increasing the complexity of care. While surgery remains the first treatment recommended for a number of patients with early breast cancer, in an increasing proportion of cases, chemotherapy is recommended as the initial treatment. There are many advantages of this approach, including down-staging of the tumour to facilitate surgery, and gaining of prognostic information based on response to chemotherapy.

Surgical techniques have improved with the evolution of oncoplastic surgery, developments in breast reconstruction techniques, and a more conservative approach to surgery for the axilla. Further advances in radiotherapy, chemotherapy and targeted therapies have improved treatment, and adjuvant endocrine therapy has a key role in the treatment of oestrogen receptor-positive (ER-positive) cancer. Gene expression profiling of primary tumours can help to guide medication treatment.

The general practitioner (GP) has an important role at every stage of the patient's breast cancer journey. This article discusses some of the recent key changes in the approach to breast cancer management, with a focus on the role of the GP.

\section{Treatment sequencing}

Breast cancer was among the first tumour types for which multidisciplinary care became routine and it is now well established as the standard of care. ${ }^{4}$ Multidisciplinary care is increasingly important as breast cancer treatment becomes more complex. The multidisciplinary team includes medical, surgical and radiation oncologists and other medical specialists such as pathologists, radiologists and genetic oncologists. In addition, the roles of the breast care nurse and allied health professionals including psychologists, physiotherapists and lymphoedema therapists are key. The team should include regular liaison with the GP to ensure comprehensive and continuous care and to provide optimal support to the patient.

The traditional treatment paradigm of surgery followed by chemotherapy followed by radiotherapy is often challenged. Different sequencing allows treatment to be individualised, tailoring it to the tumour biology and the patient's unique situation and preferences. An example of this is offering chemotherapy first ('neoadjuvant chemotherapy'; NACT). Research has classified breast cancer into five intrinsic subtypes on the basis of molecular testing (luminal A, luminal B, HER2 positive, 'basal-like' and 'normallike' $)^{5}$ and this can help guide treatment. Each type is associated with a particular phenotype and has a distinct behaviour, risk factors and sensitivity to systemic therapy that help in the selection of the appropriate regimens and medications. 


\section{Neoadjuvant chemotherapy}

Chemotherapy for breast cancer may be adjuvant (after surgery) or neoadjuvant (before surgery). In the past, NACT was reserved for cases of locally advanced, inoperable or inflammatory breast cancer. More recently, benefits of NACT have been seen in early operable breast cancer. This approach is endorsed in guidelines from Cancer Australia ${ }^{6}$ and international bodies. ${ }^{7}$ Trials have shown similar survival for adjuvant versus NACT; ${ }^{8}$ however, a major advantage of NACT is that it has prognostic significance. If NACT is given and no residual cancer is found during subsequent surgery, the patient is said to have a 'pathological complete response (pCR)' and survival is significantly improved when compared with a patient who does not achieve a pCR..$^{8,9}$ If a pCR is not achieved, there is an opportunity to give additional chemotherapy after surgery. The likelihood of a pCR is highest for tumours that are oestrogen, progesterone and HER2-receptor negative (triple negative) or are HER2 positive. ${ }^{9,10}$ When these features are seen on a diagnostic core biopsy, especially in a younger woman, NACT is likely to be considered even for a small tumour. A disadvantage of NACT is that information on lymph node status and tumour biology that would be available after up-front surgery is not available until after chemotherapy.

However, the benefit of 'in vivo' testing of chemotherapy response outweighs this in appropriately selected cases.

Other advantages of NACT are that tumour shrinkage reduces the need for mastectomy and for axillary lymph node dissection, ${ }^{10}$ allows time for genetic testing and surgical planning in the setting of a strong family history and allows more options for breast reconstruction when mastectomy is required. Patients will often seek advice from their GP after a recommendation for NACT as the sequencing may differ from their expectation of surgery first. It is acknowledged that access to NACT may be more difficult for women in rural areas.

\section{Surgery for early breast cancer}

Breast conservation surgery (wide local excision) and mastectomy remain the two surgical options for early breast cancer. When a tumour is small and is localised to one part of the breast, breast conservation is usually offered. When a tumour is larger or has multiple foci that involve two or more quadrants of the breast, mastectomy is usually recommended. There is, however, a wide range of possibilities within these two broad options. Oncoplastic surgery techniques have allowed breast conservation for larger tumours and a range of reconstructive options are available following mastectomy. In addition, women are increasingly choosing to have surgery on their contralateral (unaffected) breast for example, breast reduction surgery to obtain better symmetry or contralateral prophylactic mastectomy to reduce future risk, reduce fear of cancer recurrence and allow bilateral reconstruction.

\section{Oncoplastic breast surgery and breast reconstruction}

Most newly qualified breast cancer surgeons are now trained as 'oncoplastic' surgeons. Breast oncoplastic surgery combines the principles of cancer surgery and plastic surgery. This aims to provide adequate surgical margins for breast cancers with closure techniques that help preserve the shape and contour of the breast, aiming for the best possible cosmetic outcome. Oncoplastic surgery often allows a larger volume of breast tissue (relative to the size of the breast) to be excised and repaired and this means that larger tumours can be removed without the need for mastectomy. Therapeutic breast reduction surgery (cancer excised as part of a breast reduction-style operation) and procedures on the contralateral breast to obtain symmetry are examples of advanced oncoplastic techniques. A 2014 meta-analysis found long-term outcomes of oncoplastic surgery to be comparable to standard breast conservation surgery in terms of survival, and superior in terms of the need for margin re-excision. ${ }^{11}$

Oncoplastic surgeons are also trained in a range of post-mastectomy breast reconstruction techniques. These include skin- and nipple-sparing mastectomy with direct-to-implant reconstruction. These allow most cases of mastectomy and implant reconstruction to be completed in a single operation, without the need for tissue expansion and second-stage surgery.

Rates of breast reconstruction in Australia remain very low overall, with only around $18 \%$ of women undergoing reconstruction after a mastectomy. ${ }^{12}$ In specialist units, rates of up to $80 \%$ are reported. ${ }^{13,14}$ Not all women will choose or be medically suitable for immediate breast reconstruction; it is estimated that around $50 \%$ will take up the option when it is available. ${ }^{13}$ The very low rates of reconstruction in Australia, along with consumer survey data, ${ }^{15}$ strongly suggest that not all women are given the option. This may be largely due to referral patterns where women are not seen by specialist breast surgeons who are able to perform reconstruction or willing to refer to a multidisciplinary team colleague who can. Given the proven quality of life benefits to reconstruction for women who choose it, ${ }^{16,17}$ it is essential that women have the opportunity for a balanced discussion about breast reconstruction when mastectomy is needed or chosen, and that this discussion happens before surgery. GPs can play a part in facilitating this through appropriate referral at the time of diagnosis. Delayed breast reconstruction is also an option for most women who have had simple mastectomy.

\section{Axillary staging and management}

Assessing whether cancer has spread to the axillary lymph nodes is a key part of breast cancer staging. As most women with early breast cancer do not have lymph node metastases, minimising the long-term side effects of axillary surgery is essential. The majority of cases of early breast cancer can be managed with sentinel lymph node biopsy (SLNB) alone. This means that most women can avoid full axillary lymph node dissection (ALND) and the associated risk of long-term lymphoedema, neuralgia and shoulder stiffness. When SLNB shows no metastatic disease or shows low-volume 'micrometastases', no further surgery is indicated. ${ }^{18-20}$ When SLNB shows 1-2 positive nodes, completion 
ALND or axillary radiotherapy may be recommended. ${ }^{19}$ Trials are examining whether observation rather than further treatment is safe in this group of women, provided the primary tumour in the breast has low-risk features.

The increasing use of NACT chemotherapy has raised new questions about management of the axilla in this setting. It is now accepted practice to recommend SLNB alone for women who undergo NACT and have no clinical or radiological evidence of nodal disease pre-chemotherapy. The role of SLNB for women who are clinically node-positive pre-chemotherapy but have no detectable abnormal lymph nodes post-chemotherapy is being investigated. At present, ALND or a more localised operation called 'targeted axillary dissection' are options. ${ }^{21}$

\section{Adjuvant chemotherapy and targeted therapy}

The standard of care for many decades was to give chemotherapy after surgery, and this is still the more common sequence. Following surgery, the multidisciplinary team examines the pathology results and makes a recommendation for or against chemotherapy based on tumour factors such as tumour grade and size; receptor status for oestrogen, progesterone and HER2; and markers of proliferation including Ki67 and mitotic index. Lymph node involvement is also considered.

In some situations, further information is obtained from gene expression profiling tests. ${ }^{22,23}$ These tests use a sample of tumour to provide information about a range of genes expressed by the tumour and this can help to guide chemotherapy choices, especially in women with node-positive, ER-positive cancer. These tests are able to provide information on prognosis as well as providing information that predicts the response of the tumour to chemotherapy. These tests are used frequently in the USA but much less often in Australia because of their high cost. In Australia, gene expression profiling tends to be reserved for cases where standard histopathology leaves uncertainty as to the benefit of chemotherapy and the patient is able to fund the test.
One of the important side effects of chemotherapy is premature ovarian insufficiency in pre-menopausal women. For women wishing to preserve fertility, pre-chemotherapy referral to a fertility specialist is recommended to discuss the option of oocyte or embryo cryopreservation. In addition, it is now recommended that all pre-menopausal women have treatment with a gonadotropin-releasing hormone $(\mathrm{GnRH})$ agonist during chemotherapy (regardless of their wishes for future pregnancy) as this has been shown to protect against premature ovarian failure. ${ }^{24,25}$

\section{Targeted therapy for HER2-positive cancer}

Some breast cancer cells have an over-expression or amplification of a protein called human epidermal growth factor receptor 2 (HER2) on their surface, which makes them divide and grow aggressively (HER2 positive). Blocking HER2 receptors in HER2-positive tumours will inhibit cancer cells from growing. Trastuzumab is a monoclonal antibody that is used for patients with HER2 amplification or overexpression in breast cancer, which improves the disease-free survival and overall survival when used concomitantly with chemotherapy (adjuvant or NACT) and continued for at least one year, continuing after completion of all chemotherapy. ${ }^{26}$ The impressive improvement in survival is at the cost of cardiac toxicity, especially when trastuzumab is used in combination with anthracycline chemotherapy. ${ }^{27}$ In one study, the addition of pertuzumab (another monoclonal antibody directed against the HER2 receptor) to trastuzumab resulted in better disease-free survival in trials; however, diarrhoea was a significant side effect. ${ }^{28}$

\section{Radiotherapy}

Radiotherapy remains a key part of breast cancer treatment. In cases of breast-conserving surgery, radiotherapy is almost always indicated, with the aim of treating any microscopic residual disease. The traditional regimen consists of daily whole-breast radiation therapy for 4-6 weeks. In most cases, this is combined with a 'boost' to the tumour bed to further reduce the rate of local recurrence. ${ }^{29}$ Accelerated partialbreast irradiation, which delivers a higher dose of radiotherapy per day to a limited (localised) volume of tissue, is an alternative to whole-breast radiation therapy in selected cases of lower-risk disease, while still maintaining acceptable breast cosmesis when compared with treating the whole breast. Indications are: women with small $(\leq 3 \mathrm{~cm})$, hormone receptor-positive, lymph node-negative tumours who are $\geq 50$ years of age. ${ }^{30}$ Another option for partial breast irradiation is intra-operative radiotherapy, where a single dose of radiotherapy is given to the tumour bed during surgery. ${ }^{31}$

As recurrence rates following breast conservation are now so low, 'de-escalation' clinical trials are investigating whether radiotherapy can safely be omitted in low-risk cases. In women $\geq 65$ years of age with node-negative, hormone receptorpositive primary tumours $<3 \mathrm{~cm}$ for whom endocrine therapy is planned, omission of radiotherapy has been found to be safe $;^{32}$ however, clinical trials are ongoing and such patients should understand that without radiotherapy, the rate of in-breast recurrence may be higher over time and thorough discussion is essential.

Following mastectomy, most women can avoid radiotherapy. There are, however, increasing indications for the use of post-mastectomy radiotherapy, including larger tumour size, young age, lymph node positivity and other high-risk tumour features. ${ }^{33}$ Post-mastectomy radiotherapy can raise challenges for breast reconstruction as it increases the rate of complications from immediate implant-based breast reconstruction. ${ }^{34}$

Radiotherapy can be associated with acute and late toxicity. Incidence and severity depend on many factors such as treatment protocols and individual patient sensitivity. Long-term toxicities include breast pain, fibrosis, cardiac toxicity, lung fibrosis (after 6-12 months) and angiosarcoma (after $5-15$ years). 


\section{Adjuvant endocrine therapy}

Tamoxifen has long been used as a

five-year course of adjuvant therapy for women with ER-positive breast cancer. It is effective in both premenopausal and postmenopausal women as it lowers the risk of recurrence and mortality. More recent trials have shown an advantage of 10 years of treatment with tamoxifen over five years, so extended therapy may be recommended for women with higher-risk tumours (higher grade and/or lymph node positive)..$^{35}$

Tamoxifen is associated with a variety of side effects, with hot flushes, vaginal discharge, decreased libido and mood disturbance the most common complaints. ${ }^{36}$ A small absolute increase in the risks of thromboembolism and endometrial cancer are seen in older postmenopausal women. ${ }^{37}$ Caution must be used when combining tamoxifen with selective serotonin reuptake inhibitor antidepressants as some of these inhibit CYP2D6, which is required to convert tamoxifen to endoxifen, its active metabolite. Fluoxetine, duloxetine, bupropion and paroxetine should be avoided in women taking tamoxifen. ${ }^{38}$

Aromatase inhibitors are the alternative endocrine therapies for ER-positive tumours. These medications (letrozole, exemestane and anastrozole) are only effective for postmenopausal women. They are more effective than tamoxifen and again there is a benefit of 10 years over five years of treatment. ${ }^{39}$ Unfortunately, aromatase inhibitors are relatively poorly tolerated and can have a significant negative impact on quality of life, with side effects such as vasomotor symptoms, mood disturbance, hot flushes, vaginal dryness, musculoskeletal symptoms, cardiovascular risk and hypercholesterolaemia. Osteoporosis and fracture are serious adverse effects, and bone density must be monitored in women taking aromatase inhibitors. ${ }^{40}$ These medications are effective in reducing the risk of tumour recurrence; however, discontinuation of treatment is common because of their effect on quality of life. GPs can play a major part in encouraging women to continue their endocrine therapy and to provide symptomatic management for side effects such as joint symptoms and hot flushes (Table 1).
Ovarian function suppression using $\mathrm{GnRH}$ agonist or luteinising hormonereleasing hormone analogues are another endocrine therapy option for premenopausal women with ER-positive tumours. A recent recommendation is to offer ovarian function suppression with tamoxifen or aromatase inhibitors for women or who remain premenopausal after chemotherapy if they have tumours at higher risk of recurrence. ${ }^{41}$

\section{Conclusion}

Breast cancer treatment continues to evolve. While surgery, chemotherapy, radiotherapy and endocrine therapy are still the main treatments, there have been changes in the sequencing of treatment and advances in each therapy. Key changes include the increasing use of NACT, the development of oncoplastic surgery techniques and the recommendation for extended adjuvant endocrine therapy for up to 10 years. Being familiar with the current management of breast cancer will help GPs to support their patients and to communicate with specialists in the multidisciplinary team.

\section{Authors}

Ghaith Bahjat Heilat MD, MS, Jordanian Board of General Surgery (JBGS), Breast Surgery Fellow, Westmead Breast Cancer Institute, Westmead Hospital, NSW; Clinical Lecturer, School of Medicine/ Clinical Sciences, Faculty of Medicine, Yarmouk University, Irbid, Jordan. g.heilat@yahoo.com Meagan E Brennan FRACGP, FASBP, PhD, Staff Specialist, Westmead Breast Cancer Institute,
Westmead Hospital, NSW; Clinical Associate Professor, Northern and Western Clinical Schools, School of Medicine, Faculty of Medicine and Health University of Sydney, NSW

James French FRCAS, Head of Breast Surgery, Westmead Breast Cancer Institute, Westmead Hospital, NSW; Clinical Associate Professor, Westmead Clinical School, School of Medicine, Faculty of Medicine and Health, University of Sydney, NSW.

Competing interests: None.

Funding: None.

Provenance and peer review: Not commissioned, externally peer reviewed.

\section{References}

1. Australian Institute of Health and Welfare. Australian cancer incidence and mortality books: Breast cancer. Canberra: AlHW, 2018.

2. Australian Institute of Health and Welfare. Breast cancer in Australia: An overview. Cancer series no. 71. Cat. no. CAN 6. Canberra: AlHW, 2012.

3. Australian Institute of Health and Welfare. Cancer in Australia 2001. Cat. no. CAN 23. Canberra: AlHW, 2004.

4. National Breast Cancer Centre. Multidisciplinary care in Australia: A national demonstration project in breast cancer. Camperdown, NSW: NBCC, 2003.

5. Prat A, Parker JS, Karginova O, et al. Phenotypic and molecular characterization of the claudin-low intrinsic subtype of breast cancer. Breast Cancer Res 2010;12(5):R68. doi: 10.1186/bcr2635.

6. Cancer Australia. Cancer Australia statement: Influencing best practice in breast cancer. Surrey Hills, NSW: Cancer Australia, 2016.

7. The American Society of Breast Surgeons. Performance and practice guidelines for the use of neoadjuvant systemic therapy in the management of breast cancer. Columbia, MD: The American Society of Breast Surgeons, 2018.

8. Mauri D, Pavlidis N, loannidis JP. Neoadjuvant versus adjuvant systemic treatment in breast cancer: A meta-analysis. J Natl Cancer Inst 2005;97(3):188-94. doi: 10.1093/jnci/dji021.

9. Symmans WF, Wei C, Gould R, et al. Long-term prognostic risk after neoadjuvant chemotherapy associated with residual cancer burden and breast cancer subtype. J Clin Oncol 2017;35(10):1049-60. doi: 10.1200/JCO.2015.63.1010.

\section{Table 1. Aromatase inhibitors: Common side effects and management}

\begin{tabular}{ll}
\hline Side effect & Management \\
\hline Musculoskeletal & $\cdot$ Physical exercise \\
\hline Vaginal dryness & NSAIDs \\
\hline Hot flushes & $\cdot$ Vaginal moisturisers and lubricants \\
\hline Osteoporosis and fracture & $\cdot$ SSRls \\
& $\cdot$ Gabapentin \\
& $\cdot$ Calcium \\
& $\cdot$ Vitamin D3 \\
& $\cdot$ Bisphosphonates
\end{tabular}

NSAID, non-steroidal anti-inflammatory drug; SSRI, selective serotonin reuptake inhibitor 
10. King T, Morrow M. Surgical issues in patients with breast cancer receiving neoadjuvant chemotherapy. Nat Rev Clin Oncol 2015;12(6):335-43. doi: 10.1038/ nrclinonc.2015.63.

11. Losken A, Dugal CS, Styblo TM, Carlson GW. A meta-analysis comparing breast conservation therapy alone to the oncoplastic technique. Ann Plast Surg 2013;72(2):145-49. doi: 10.1097/ SAP.0b013e318260559.

12. Flitcroft K, Brennan M, Costa D, Spillane A Documenting patterns of access to breast reconstruction in Australia: The national picture. Breast 2016;30:47-53. doi: 10.1016/j. breast.2016.08.013.

13. Brennan ME, Spillane AJ. Uptake and predictors of post-mastectomy reconstruction in women with breast malignancy: Systematic review. Eur J Surg Oncol 2013;39(6):527-41. doi: 10.1016/j.ejso.2013.02.021.

14. Wong A, Snook K, Brennan M, et al. Increasing breast reconstruction rates by offering more women a choice. Breast 2014;84(1-2):31-6. doi: 10.1111/ans.12471.

15. Breast Cancer Network Australia. The BEACON. Issue of concern: Breast reconstruction. Issue 63. Camberwell, Vic: BCNA, 2013.

16. Chen W, Lv X, Xu X, Gao X, Wang B. Metaanalysis for psychological impact of breast reconstruction in patients with breast cancer. Breast Cancer 2018;25(4):464-69. doi: 10.1007/ s12282-018-0846-8.

17. Fanakidou I, Zyga S, Alikari V, Tsironi M, Stathoulis J, Theofilou P. Mental health, loneliness, and illness perception outcomes in quality of life among young breast cancer patients after mastectomy: The role of breast reconstruction. Qual Life Res 2018; 27(2):539-43. doi: 10.1007/ s11136-017-1735-x

18. Galimberti V, Cole BF, Zurrida S, et al. Axillary dissection versus no axillary dissection in patients with sentinel-node micrometastases (IBCSG 23-01): A phase 3 randomised controlled trial. Lancet Oncol 2013;14(4):297-305. doi: 10.1016/ S1470-2045(13)70035-4.

19. Donker M, van Tienhoven G, Straver ME, et al. Radiotherapy or surgery of the axilla after a positive sentinel node in breast cancer (EORTC 10981-22023 AMAROS): A randomised, multicentre, open-label, phase 3 non-inferiority trial. Lancet Oncol 2014;15(12):1303-10. doi: 10.1016/S1470-2045(14)70460-7.

20. Giuliano AE, Ballman KV, McCall L, et al. Effect of axillary dissection vs no axillary dissection on 10-year overall survival among women with invasive breast cancer and sentinel node metastasis: The ACOSOG Z0011 (Alliance) randomized clinical trial. JAMA 2017;318(10):918-96. doi: 10.1001/ jama.2017.11470.

21. Caudle AS, Yang WT, Mittendorf EA, et al. Selective surgical localization of axillary lymph nodes containing metastases in patients with breast cancer: A prospective feasibility trial. JAMA Surg 2015;150(2):137-43. doi: 10.1001/ jamasurg.2014.1086.

22. Mamounas EP, Russell CA, Lau A, Turner MP Albain KS. Clinical relevance of the 21-gene Recurrence Score ${ }^{\circledast}$ assay in treatment decisions for patients with node-positive breast cancer in the genomic era. NPJ Breast Cancer 2018;4(27):1-5. doi: 10.1038/s41523-018-0082-6.

23. Jensen M-B, Lænkholm A-V, Nielsen TO, et al. The Prosigna gene expression assay and responsiveness to adjuvant cyclophosphamide- based chemotherapy in premenopausal high-risk patients with breast cancer. Breast Cancer Res 2018;20:79. doi: 10.1186/s13058-018-1012-0.

24. Cui W, Stern CJ, Hickey M, Goldblatt F. Preventing ovarian failure associated with chemotherapy. Med J Aust 2018;209(9):412-16. doi: 10.5694/ mja18.00190.

25. Lambertini M, Richard F, Nguyen B, Viglietti G Villareal-Garza C. Ovarian function and fertility preservation in breast cancer: Should gonadotropin-releasing hormone agonist be administered to all premenopausal patients receiving chemotherapy? Clin Med Insights Reprod Health 2019;13:1179558119828393. doi: 10.1177/1179558119828393.

26. Moja L, Tagliabue L, Balduzzi S, et al. Trastuzumab containing regimens for early breast cancer. Cochrane Database Syst Rev 2012:4:CD006243. doi: 10.1002/14651858.CD006243.pub2.

27. Banke A, Fosbøl EL, Ewertz M, et al. Long-term risk of heart failure in breast cancer patients after adjuvant chemotherapy with or without trastuzumab. JACC Heart Fail 2019;7(3):217-24. doi: 10.1016/j.jchf.2018.09.001.

28. Von Minckwitz G, Procter M, de Azambuja E, et al. Adjuvant pertuzumab and trastuzumab in early HER2-positive breast cancer. N Engl J Med 2017:377:122-31. doi: 10.1056/NEJMoa1703643.

29. Bartelink $H$, Maingon $P$, Poortmans $P$, et al. Whole-breast irradiation with or without a boost for patients treated with breast-conserving surgery for early breast cancer: 20-year follow-up of a randomised phase 3 trial. Lancet Oncol 2015;16(1):47-56. doi: 10.1016/S14702045(14)71156-8.

30. Polgár C, Ott OJ, Hildebrandt G, et al. Late sideeffects and cosmetic results of accelerated partial breast irradiation with interstitial brachytherapy versus whole-breast irradiation after breastconserving surgery for low-risk invasive and in-situ carcinoma of the female breast: 5 -year results of a randomised, controlled, phase 3 trial. Lancet Oncol 2017:18(2):259-68. doi: 10.1016/ S1470-2045(17)30011-6.

31. Njeh CF, Saunders MW, Langton CM. Accelerated partial breast irradiation (APBI): A review of available techniques. Radiat Oncol 2010;5:90. doi: 10.1186/1748-717X-5-90.

32. Hughes KS, Schnaper LA, Bellon JR, et al. Lumpectomy plus tamoxifen with or without irradiation in women age 70 years or older with early breast cancer: Long-term follow-up of CALGB 9343. J Clin Oncol 2013;31(19):2382-87. doi: 10.1200/JCO.2012.45.2615.

33. Remick J, Amin NP. Radiation therapy, breast cancer, postmastectomy. 2018 Aug 2. StatPearls [Internet]. Treasure Island (FL): StatPearls Publishing; 2018.

34. El-Sabawi B, Ho AL, Sosin M, Patel KM. Patientcentered outcomes of breast reconstruction in the setting of post-mastectomy radiotherapy: A comprehensive review of the literature. J Plast Reconstr Aesthet Surg 2017;70(6):768-80. doi: 10.1016/j.bjps.2017.02.015

35. Francis PA. Adjuvant endocrine therapy for premenopausal women: Type and duration. Breast 2017;34(Suppl 1):S108-S11. doi: 10.1016/j. breast.2017.06.040

36. Frechette $D$, Paquet $L$, Verma $S$, et al. The impact of endocrine therapy on sexual dysfunction in postmenopausal women with early stage breast cancer: Encouraging results from a prospective study. Breast Cancer Res Treat 2013;141(1):111-17. doi: 10.1007/s10549-013-2659-y.
37. Davies C, Godwin J, Gray R, et al. Relevance of breast cancer hormone receptors and other factors to the efficacy of adjuvant tamoxifen: Patientlevel meta-analysis of randomised trials. Lancet 2011;378(9793):771-84. doi: 10.1016/S01406736(11)60993-8.

38. Juurlink D. Revisiting the drug interaction between tamoxifen and SSRI antidepressants. BMJ 2016;354:i5309. doi: 10.1136/bmj.i5309.

39. Early Breast Cancer Trialists' Collaborative Group (EBCTCG). Aromatase inhibitors versus tamoxifen in early breast cancer: Patient-level meta-analysis of the randomised trials. Lancet 2015;386(10001):1341-52. doi: 10.1016/S01406736(15)61074-1.

40. Amir E, Seruga B, Niraula S, Carlsson L, Ocaña A. Toxicity of adjuvant endocrine therapy in postmenopausal breast cancer patients: A systematic review and meta-analysis. J Natl Cancer Inst 2011;103(17):1299-309. doi: 10.1093/jnci/djr242.

41. Coates AS, Winer EP, Goldhirsch A, et al. Tailoring therapies - improving the management of early breast cancer: St Gallen International Expert Consensus on the Primary Therapy of Early Breast Cancer 2015. Ann Oncol 2015;26(8):1533-46. doi: 10.1093/annonc/mdv221. 